\title{
Use of Palmaz Stents in a Newborn With Congenital Aneurysms and Coarctation of the Abdominal Aorta
}

\author{
J. Ballweg, MD, R. Liniger, BS, A. Rocchini, MD, and R. Gajarski, ${ }^{*}$ MD \\ Anomalies of the abdominal aorta are rare in the pediatric population limiting the \\ reported knowledge base from which management decisions can be made. A 3-week- \\ old male with congenital abdominal aortic coarctation and multiple aneurysms pre- \\ sented with malignant hypertension. We report the safe deployment of overlapping \\ Palmaz stents using a 4-French catheter delivery system with significant relief of the \\ coarctation gradient and restoration of adequate renal perfusion. 02006 Wiley-Liss., Inc.
}

Key words: abdominal coarctation; stents; interventional catheterization

\section{INTRODUCTION}

Among pediatric patients, the most common etiology for coarctation of the abdominal aorta is Takayasu's arteritis, which, rarely, can also accompany aneurysms. In the absence of coarctation, abdominal aortic aneurysms are often associated with connective tissue disease, umbilical artery catheterization, and infectious or vasculitic processes. Multiple congenital abdominal aortic aneurysms are exceedingly rare with only five cases reported in live infants [1-5]. Uncontrolled hypertension from chronic renin-angiotensin activation, gastrointestinal tract ischemia, renal failure, aneurysm rupture, cardiac hypertrophy, and arrhythmias are potentially life threatening sequelae of untreated disease. Abdominal coarctation, as the primary source of clinical pathology, in conjunction with multiple aneurysms has not been previously reported and presents a uniquely challenging management problem. Interventional catheterization techniques utilizing stents to relieve coarctation of the aorta [6-8] as well as surgical repairs have been previously described in older children and adults [9-12]. There are currently no reports describing the use of stents for abdominal coarctation in newborns or infants, likely related to the previous lack of an appropriately sized delivery system and the limited potential for adequate stent redilation with somatic growth. Our patient was a 3-week-old with both congenital aneurysms and abdominal coarctation involving the renal arteries. To our knowledge, this is the first reported neonate with concomitant congenital abdominal aneurysms and coarctation successfully treated with Palmaz stents.

\section{CASE REPORT}

A term male weighing $2.93 \mathrm{~kg}$ born to a healthy 34year-old G2P1 woman at an outside hospital was known to have an aortic aneurysm by prenatal ultrasound. At 2 weeks of age, his primary care physician found him to be hypertensive with an upper extremity blood pressure of $185 / 135 \mathrm{~mm} \mathrm{Hg}$, after which he was admitted to the local neonatal ICU. Angiography demonstrated multiple abdominal aortic aneurysms involving the right iliac artery as well as a hypoplastic right renal artery. No family history of early renal failure, dialysis, need for renal transplant, malignant hypertension, Marfan's Disease, Ehlers-Danlos, or other connective tissue disorders was elicited.

The infant was transferred to the University of Michigan at 3 weeks of age on nitroprusside and esmolol infusions to maintain systolic blood pressures of $100-110 \mathrm{~mm} \mathrm{Hg}$ to ensure adequate renal perfusion. Additionally, heparin anticoagulation had been initiated prior to transfer following detection of aortic aneurysm thrombus. On admission, right upper extremity blood pressure was $94 / 53 \mathrm{~mm} \mathrm{Hg}$ and left lower extremity blood pressure was $33 \mathrm{~mm} \mathrm{Hg}$ systolic (no diastolic as this was measured by Doppler probe). Additional physical exam revealed the presence of posteriorly rotated ears, mild micrognathia, a 2/6 systolic murmur radiating to back, midline bruit (no epigastic pulsations noted),

Division of Pediatric Cardiology, Department of Pediatrics, University of Michigan, Ann Arbor, Michigan

*Correspondence to: R. Gajarski. C.S. Mott Children's Hospital, University of Michigan Health System, L1214 Women's / Box 0204, 1500 East Medical Center Drive, Ann Arbor, MI 48109.

E-mail: rjgaj@umich.edu

Received 4 May 2005; Revision accepted 10 February 2006

DOI 10.1002/ccd.20749

Published online 12 September 2006 in Wiley InterScience (www. interscience.wiley.com). 


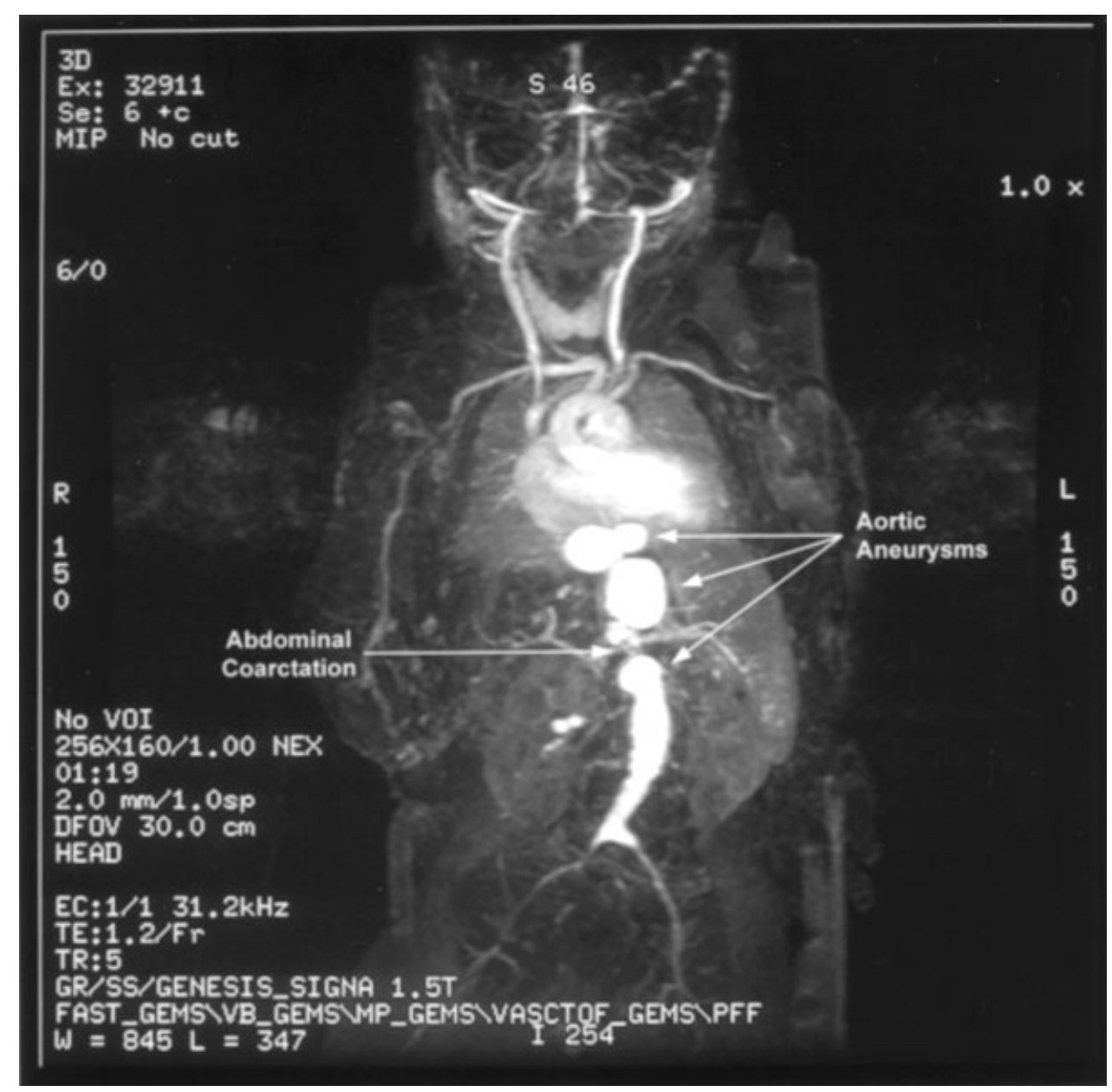

Fig. 1. Coronal view MRI image demonstrates the location of the lower thoracic and abdominal aortic coarctations (arrows) near the level of the diaphragm with intersegment coarctation (arrow). Note that the renal and femoral arteries are hypoplastic.

weak femoral pulses, and talipes equinovarus, bilaterally. No laboratory evidence for infectious or inflammatory processes, including an ESR 11, negative blood and urine cultures and low viral antibody titers, was found. Chromosomal FISH analysis did not identify a $22 q 11$ microdeletion or other genetic anomalies. An echocardiogram revealed normal intracardiac anatomy with normal systemic and pulmonary venous return, patent foramen ovale with left to right shunting, no ventricular septal defect, moderate concentric left ventricular hypertrophy, and good biventricular function. Magnetic resonance angiography (MRA) of the brain, chest, and abdomen revealed three abdominal aortic aneurysms extending to the iliac bifurcation, as well as severe coarctation/stenosis between aneurysm segments and hypoplastic renal arteries (Fig. 1).

With this data, the baby was taken to the cardiac catheterization laboratory for further anatomic delineation by angiography and stent implantation in an effort to relieve the coarctation, improve renal blood flow, and decrease systemic hypertension. Anatomy similar to that identified by MRA was demonstrated (Fig. 2) with a peak gradient across the coarctation of $53 \mathrm{~mm}$ $\mathrm{Hg}$. Recorded dimensions included proximal descending aorta $6.3 \mathrm{~mm}$, area of coarctation $2 \mathrm{~mm}$, distal descending aorta past renal arteries $10 \mathrm{~mm}$, distal aorta at level of iliac bifurcation $6.3 \mathrm{~mm}$, proximal right femoral artery $3 \mathrm{~mm}$. There were four aneurysms, measuring $16.7 \times 17.8 \mathrm{~mm}^{2}, 11.1 \times 10.3 \mathrm{~mm}^{2}, 23.8 \times$ $19.8 \mathrm{~mm}^{2}$, and $9.8 \times 13 \mathrm{~mm}^{2}$. Using interventional techniques previously described, [13] three Palmaz Corinthian PQ124 stents were placed in an overlapping configuration through the region of abdominal coarctation followed by the placement of a Palmaz Genesis PG184 stent at the most distal aspect of narrowing via a 4-French catheter delivery system. The most proximal stent was initially inflated using a 4-mm Ranger balloon, followed by inflation with a 5-mm Tyshak II 


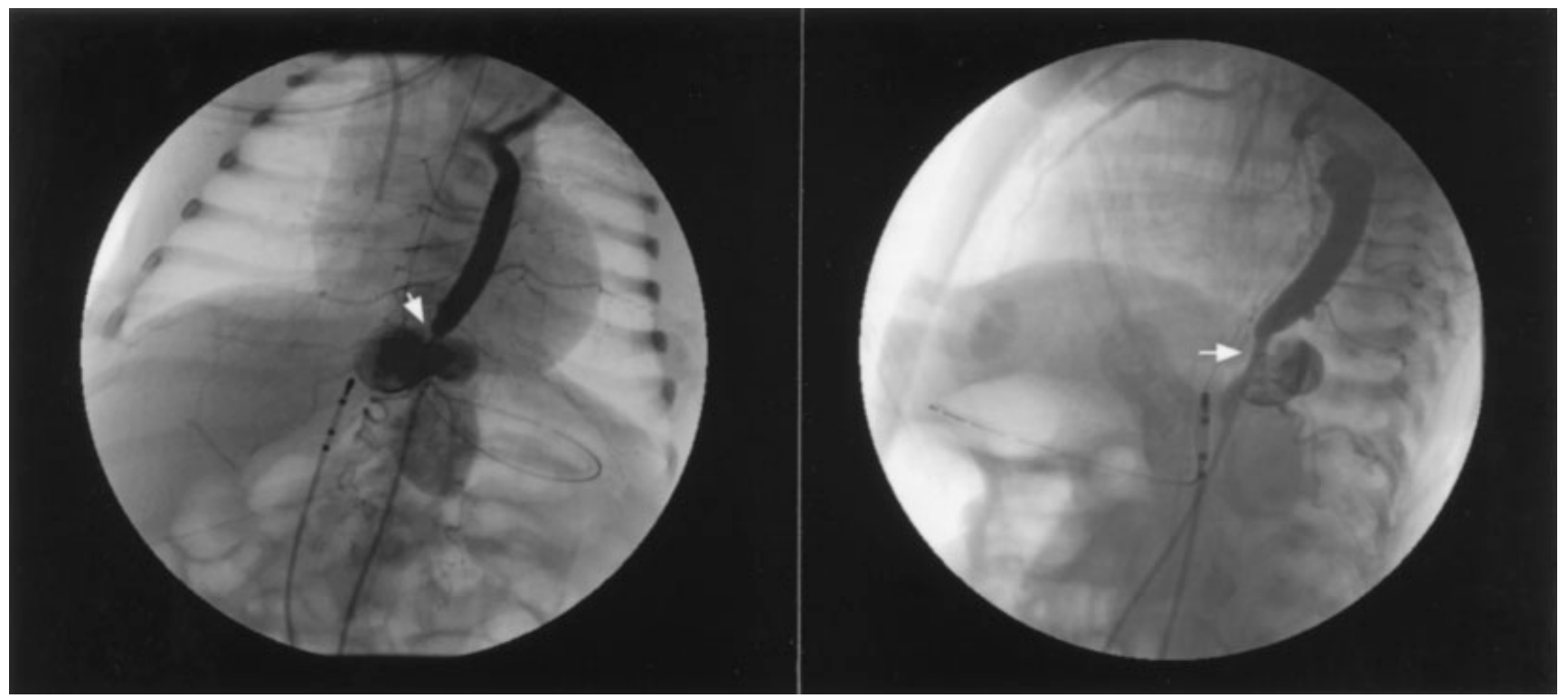

Fig. 2. AP and lateral projections of the thoracic and abdominal aorta (left and right images, respectively). Note appearance of coarctation segment (arrow) and location of aneurysms corroborate MRI image.

balloon. All of the subsequent stents were inflated using a 4-mm Ranger Balloon catheter. Following stent implantation, there was a $60 \%$ reduction in the peak systolic gradient from 53 to $21 \mathrm{~mm} \mathrm{Hg}$. Additionally, there was no evidence of aortic tear, dissection or contrast extravasation (Fig. 3). The baby tolerated the procedure well and was transferred to the cardiac intensive care unit. No femoral pulses could be palpated after return to the ICU, and they remained absent at the time of discharge from the hospital.

Following the interventional procedure, the infant maintained systolic blood pressures below $120 \mathrm{~mm} \mathrm{Hg}$ on oral amlodipine and propranolol. Because of previously noted thrombus, we chose more aggressive anticoagulation with Coumadin (target INR 1.5-2.0). He was discharged after a total hospital stay of 10 days with normal renal function.

On an antihypertensive regimen including amlodipine $4 \mathrm{mg}$ bid, labetalol $24 \mathrm{mg}$ bid, and aldactone $2.5 \mathrm{mg}$ bid, a repeat catheterization 4 months later revealed a left ventricular pressure of 124/16 mm Hg, and ascending/descending thoracic aortic pressures of $125 / 68 \mathrm{~mm}$ $\mathrm{Hg}$. The abdominal aortic pressure waveform distal to the multiple stents was non-pulsatile, with a mean pressure of $74 \mathrm{~mm} \mathrm{Hg}$. Cineangiograms demonstrated a normal appearing aorta to the level of the diaphragm. The internal lumen of the stented portion of the abdominal aorta varied from 2.5 to $3.5 \mathrm{~mm}$ in diameter. A short gap had developed between the first and second stents. All other stents remained overlapped. No excessive neointimal proliferation was identified within any of the stents. Marked ectatic changes of the abdominal aorta were recorded distal to the last stent, with an aortic diameter of $12-15 \mathrm{~mm}$. The superior mesenteric artery and renal arteries arose from this ectatic portion of the aorta. Both right and left renal arteries filled without evidence of renal artery stenosis. No thrombosis was noted within the aorta or within the saccular aneurysms.

At the time of catheterization, it was known that the infant had developed a large, retroperitoneal myofibroma, which was compressing the spinal cord at the level of L2 with resultant neurologic symptoms. He underwent resection of the tumor 2 weeks after the catheterization and was discharged.

\section{DISCUSSION}

Stent placement can be very effective in relieving arch obstruction safely and has become a frequently used alternative form of therapy in patients with native or recurrent coarctation. Stents support the integrity of the vessel wall after balloon dilation by opposing the recoil of the elastic vascular stenosis and reapplying the torn intima to the media. This minimizes the extension of wall tears and subsequent dissection or aneurysm formation that could occur after balloon angioplasty alone.

Limited data is available in regard to stent therapy in young children. In three large series, five small 


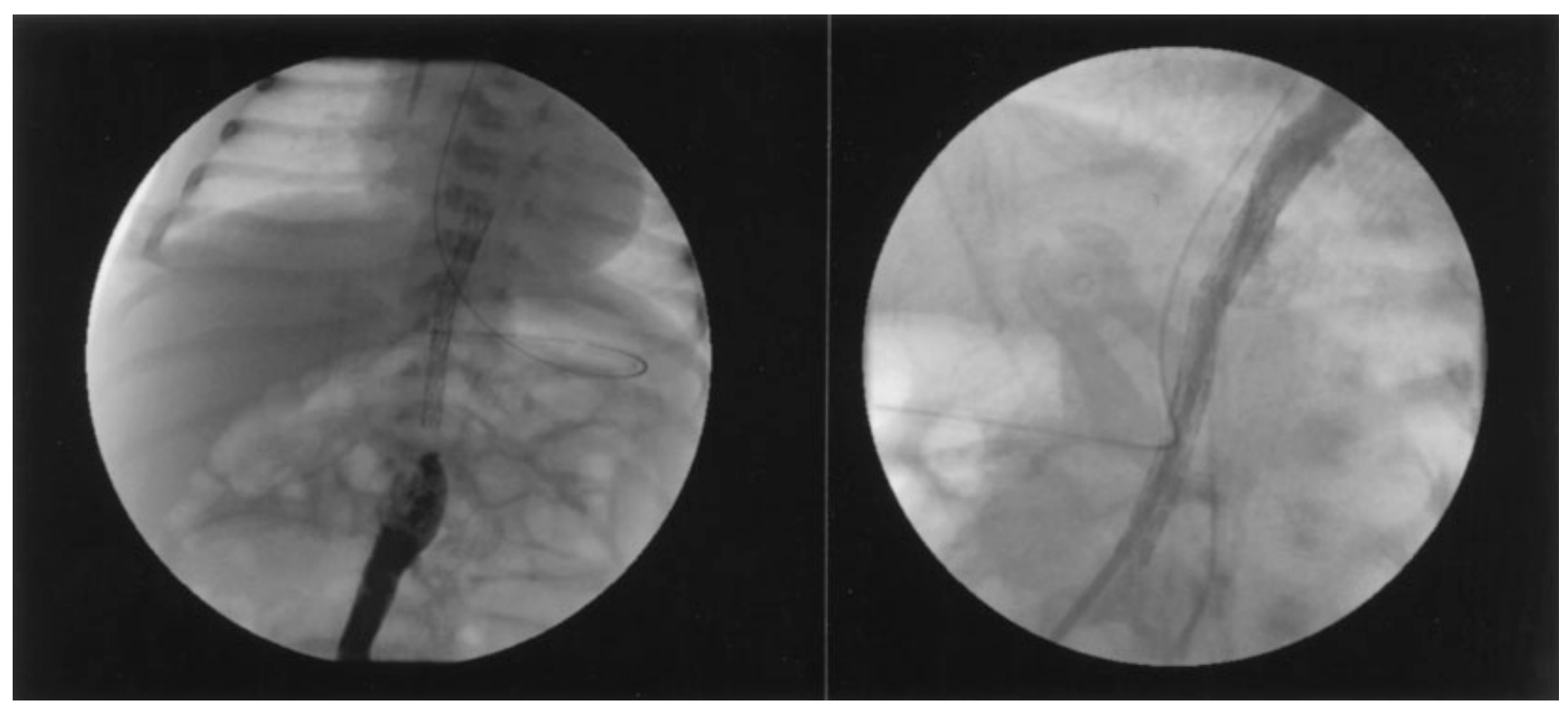

Fig. 3. AP and lateral projections of abdominal aorta (left and right images, respectively) post-stent implantation. Note the overlapping configuration of the stents and resolution of coarctation segments. The lateral image demonstrates no evidence of contrast extravasation after stent delivery.

children, including two infants, underwent stent implantation, with the majority having had previous surgical and or balloon dilation interventions. Stent implanation has also been described in a neonate who was deemed too unstable for surgical repair of thoracic aortic coarctation. Limitations to stent placement in infants and young children include the lack of small delivery systems, the higher rate of restenosis from neointimal thickening, [14] as well as the inability to place a stent whose maximal diameter will reach normal adult aortic size resulting in stent-mediated stenosis.

The use of covered stents in the setting of vascular aneurysms is well documented. The use of Palmaz stents in the setting of coarctation and saccular aneurysm in children has been described by Suarez de Lezo et al. Their findings suggest that the blood column favored by the stent tube could partially isolate the covered sac from the bloodstream, enabling exclusion or an inhibition of growth with advancing age [15]. Alternatively, coil obliteration of the cavity excluding the aneurysm completely from the blood surface has also been demonstrated.

This case demonstrates an infant with multiple areas of abdominal coarctation in association with congenital aneurysms. The arch obstruction was adequately relieved by stent placement. Multiple aneurysms were isolated as well by the serial, overlapping stents. Thrombus, which was initially noted within the aneurysms subsequently resolved after stent placement and anticoagula- tion. The hypertension has been adequately controlled with relief of the arch obstruction in conjunction with antihypertensive agents. Renal blood flow has been preserved with the current therapies. Renal revascularization may become necessary if the renal arteries become stenotic or obstructed by the aortic stents or if hypertension cannot be reasonably controlled by oral antihypertensive medications. Finally, redilatation of the stents will be necessary to maintain the abdominal aorta free of obstruction. Unfortunately, the stents utilized in this case can only safely be redilated to a maximum diameter of $\sim 11 \mathrm{~mm}$. Since it is likely that this diameter will be inadequate with somatic growth of this infant, surgical augmentation of the aorta with prosthetic graft material (including the stented areas) will be necessary at a future time.

\section{REFERENCES}

1. Connolly JE, Wilson SE, Lawrence PL, Fuhitani RM. Middle aortic syndrome: Distal thoracic and abdominar coarctation, a disorder with multiple etiologies. J Amer Coll Surg 2002;194(6): 774-81.

2. Mehall J, Saltzman DA, Chandler JC, Kidd JN, Wells T, Smith SD. Congenital abdominal aortic aneurysm in the infant: Case report and review of the literature. J Pediatr Surg 2000;36(4):657-658.

3. Kashani IA, Sklansky MS, Movahed H, Lucas VW, Rothman A. Successful balloon dilation of an abdominal coarctation of the aorta in a patient with presumed takayasu's aortitis. Cath Cardio Diag 1996;38:406-409.

4. Howarth MB. Aneurysm of abdominal aorta in the newborn infant. N Engl J Med 1967;276:1133-1134. 
5. Latter D, Beland MJ, Batten A, Tchervenkov CI, Dobell ARC. Congenital abdominal aortic aneurysm. Can J Surg 1989;32:135-138.

6. Saad SA, May A. Abdominal aortic aneurysm in a neonate. J Pediatr Surg 1991;26:1423-1424.

7. Kim ES, Caiati JM, Tu J, Nowygrod R, Stolar CJ. Congenital abdominal aortic aneurysm causing renovascular hypertension, cardiomyopathy, and death in a 19-day-old neonate. J Pediatr Surg 2001;36(9):1445-1449.

8. Eliason JL, Passman MA. Durability of percutaneous angioplasty and stent implantation for treatment of abdominal aortic coarctation: Case report. Vasc Surg 2000;35(5):397-401.

9. Ing FF, Goldberg B, Siegel DH, Trachtman H, Bierman FZ. Arterial stents in the management of neurofibromatosis and renovascular hypertension in a pediatric patient: Case report of a new treatment modality. Cardiovasc Interven Radiol 1995;18(6): 414-418.

10. Vaccaro PS, Myers JC, Smead WL. Surgical correction of abdominal aortic coarctation and hypertension. J Vasc Surg 1986; 3(4):643-648.
11. D’Souza SJA, Tsai W, Silver MM, Chait P, Benson LN, Silverman E, Hebert D, Balfe JW. Diagnosis and management of stenotic aorto-arteriopathy in childhood. J Pediatr 1998;132(6): 1016-1022.

12. Mickley V, Fleiter T. Coarctations of descending and abdominal aorta: Long-term results of surgical therapy. J Vasc Surg 1998; 28(2):206-214

13. Cheatham JP. Stenting of coarctations of the aorta. Cath Cardio Interv 2001;54:112-125.

14. Thanopoulos BD, Hadjinikolaou L, Konstadopoulou GN, Tsaousis GS, Triposkiadis F, Spirou P. Stent treatment for coarctation of the aorta: Intermediate term follow up and technical considerations. Heart 2000;64:65-70.

15. Suarez de Lezo J, Pan M, Romero M, Medina A, Segura J, Lafuente M, Pavlovic D, Hernandez E, Melian F, Espada J. Immediate and follow-up findings after stent treatment for severe coarctation of aorta. Amer J Cardio 1999;83:400406. 